\title{
EFFECT OF ORGANIC MANURE WITH BIOFERTILIZERS ON GROWTH, FLOWERING AND CHEMICAL COMPONENTS OF Echinacea purpurea (L.) MOENCH PLANT
}

\author{
(Received:3.8.2010)
}

\author{
By \\ A.M.S. Arafa, H.M. Fahmy, M. A. M. Saied* and H.S.A.Mashaal * \\ Ornamental Horticulture Department, Cairo University, Giza, Egypt \\ *Medicinal and Aromatic Plant Department, National Research Centre, Dokki, Egypt
}

\begin{abstract}
The performance of organic manure with biofertilizers was compared with chemical fertilizer in two successive seasons of 2005/2006 and 2006/2007 at the experimental area of the Ornamental Horticulture Department, Faculty of Agriculture, Cairo University, Giza, Egypt to investigate the possibility of organic manure with biofertilizer utilization instead of chemical fertilizers. The obtained results indicated that vegetative, flowering and rooting growth parameters, NPK and chicoric acid content were augmented on the application of organic manure with biofertilizers especially when chicken manure + Azospirillum lipoferum + Bacillus megatherium treatment was used as compared to NPK as chemical fertilizer treatment.
\end{abstract}

Key words: Echinacea purpurea, biofertilizers, organic fertilizers.

\section{INTRODUCTION}

Echinacea purpurea (L.) Moench is a perennial medicinal herb, known as the purple coneflower, belonging to the Asteraceae family. It has gained an economic importance as a medicinal and ornamental plant. Echinacea extracts have been used by Native Americans as pain killer, snakebite treatment, and cold remedy for centuries. It has been used for its antibiotic and immunostimulatory properties (Kindscher, 1989). Using organic fertilization to increase yield and quality is more acceptable than chemical fertilizer, especially in medicinal and aromatic plants. (Abou El-Fadl et al., 1990). Also Echinacea extracts showed antioxidative, antibacterial, antiviral, antifungal properties and are used for treating common cold, respiratory and urinary diseases (Barrett, 2003).

Numerous studies using organic and biofertilizers have been undertaken to improve growth, yield and active components in anise (Safwat et al., 2001), cumin (Safwat and Badran, 2002), fennel ( Badran and Safwat, 2004 and Azzaz et al., 2009), Rosmarinus officinalis (Abd El Aziz et al., 2007) and Borago officinalis and Echium vulgare (Hendawy and El Gengaihi, 2010).

Hendawy (1995), investigated the effect of chemical fertilizers on Echinacea purpurea plant while (Rashad, 1999), studied the effect of different organic fertilizers on the same plant on growth, yield and active components. But the use of organic manure with biofertilizers has not been extensively used in Echinacea purpurea cultivation. Therefore, the objective of this study was to evaluate the effect of organic manures with biofertilizers in comparison with chemical fertilizers on growth, flowering and chemical components of Echinacea purpurea plant.

\section{MATERIALS AND METHODS}

Two successive field experiments were established during seasons of 2005/2006 and 2006/2007, at the Experimental Area of the Ornamental Horticulture Department, Faculty of Agriculture, Cairo University, Giza, Egypt, to study the influence of organic manure with biofertilizers in comparison with chemical fertilizers on growth, NPK, and chicoric acid content of Echinacea purpurea (L.) Moench plant.

\subsection{Seed source}

A seed lot was obtained from the United States of America.

\subsection{Cultivation}

Seeds of Echinacea purpurea were sown inside the greenhouse in clay soil on the first of November. The seedlings were transplanted to the field on the first of April 2006 and 2007 in both seasons, respectively. 
The area of the experimental plot was $4 \mathrm{~m}^{2}$ consisted of 3 rows, the planting distance was 30 $\mathrm{cm}$ apart and each plot contained 18 plants.

\subsection{The soil used}

The physical and chemical characteristics of the soil used are presented in Table (1), and chemical analyses of the organic manures are shown in Table (2).
Biofertilizers were kindly obtained from The Department of Microbiology, National Research Center.

Treatments were as follows:

1-Control (NPK recommended doses).

2- FYM + Azospirillum lipoferum.

3- FYM+ Bacillus megatherium.

4- FYM+ Azospirillum lipoferum + Bacillus

Table (1): Some physical and chemical characteristics of the soil of the experimental field during the two seasons of 2005/2006 and 2006/2007

\begin{tabular}{|l|c|c|c|c|c|c|c|c|c|c|c|}
\hline & \multirow{2}{*}{ Texture } & pH & E.c.(ds/m) & $\mathbf{C a C O}_{3}$ & $\%$ & \multicolumn{3}{|c|}{$\begin{array}{c}\text { Available } \\
\text { nutrients (ppm) }\end{array}$} & \multicolumn{3}{|c|}{ Water soluble ions (meq/l) } \\
\cline { 5 - 12 } & & & $\begin{array}{l}\text { Total } \\
\mathbf{N}\end{array}$ & $\mathbf{P}$ & $\mathbf{K}$ & $\mathbf{C a}$ & $\mathbf{M g}$ & $\mathbf{H C O}$ & $\mathbf{C L}$ & $\mathbf{S O}_{\mathbf{4}}$ \\
\hline $\begin{array}{l}\text { Sand } \\
\text { Loam }\end{array}$ & 8.2 & 2.3 & 2.9 & 473 & 35 & 31.4 & 12 & 7.9 & 4.1 & 10 & 21.3 \\
\hline
\end{tabular}

Table (2): Some physical and chemical properties of the organic manure during the two seasons of 2005/2006 and 2006/2007:

\begin{tabular}{|c|c|c|c|c|c|c|c|}
\hline $\begin{array}{c}\text { Organic } \\
\text { manure } \\
\text { type }\end{array}$ & $\mathbf{p H}$ & $\begin{array}{c}\text { Ec } \\
\text { m.mohs }\end{array}$ & $\begin{array}{c}\text { Organic } \\
\text { matter }(\%)\end{array}$ & $\begin{array}{c}\mathbf{C} / \mathbf{N} \\
\text { ratio }\end{array}$ & $\mathbf{N}(\boldsymbol{\%})$ & $\mathbf{P}(\boldsymbol{\%})$ & $\mathbf{K}(\boldsymbol{\%})$ \\
\hline FYM & 7.30 & 5.0 & 33.2 & 18.4 & 1.90 & 0.54 & 0.7 \\
\hline CH.M & 7.41 & 3.8 & 28.0 & -- & 2.46 & 0.77 & 1.4 \\
\hline Compost & 7.49 & 4.4 & 35.0 & 18.95 & 2.00 & 0.66 & 1.0 \\
\hline
\end{tabular}

FYM: Farm Yard Manure. CH.M: Chicken Manure $\quad$ Compost: Nile Compost.

\subsection{Fertilization}

Three resources of fertilization were used.

\subsubsection{Chemical fertilizers as a control treatment}

The recommended doses on Echinacea purpurea plant were added as follows:

$150 \mathrm{~kg}$ ammonium sulphate $(20.5 \% \mathrm{~N})+100 \mathrm{~kg}$ calcium superphosphate $\left(15.5 \% \mathrm{P}_{2} \mathrm{O}_{5}\right)+100 \mathrm{~kg}$ potassium sulphate $\left(48 \% \mathrm{~K}_{2} \mathrm{O}\right)$ per feddan, in three doses, the first one, month after transplanting, the second, two weeks later and the third dose, two weeks after the second one (Hendawy, 1995).

\subsubsection{Organic fertilizers}

The amounts of organic fertilizers were calculated according to their content of nitrogen which must be equal to that found in the amount of ammonium sulphate recommended as chemical fertilizer as follows:

1.62 ton Farm Yard Manure (FYM), 1.16 ton chicken manure $(\mathrm{CH} \mathrm{M})$ and 1.52 ton compost per feddan were added during soil preparation.

\subsubsection{Biofertilizers}

Bacillus megatherium (B), Azospirillum lipoferum $(A)$, and a mixture of them, $(2.5 \mathrm{~L}$ of the used strain $+22.5 \mathrm{~L}$ Tap water) / Feddan were added to the soil surface beside plants after a month from transplantation. megatherium.

5- CH.M+ Azospirillum lipoferum.

6- CH.M+ Bacillus megatherium.

7- CH.M+ Azospirillum lipoferum + Bacillus megatherium.

8- Compost + Azospirillum lipoferum.

9- Compost + Bacillus megatherium .

10-Compost + Azospirillum lipoferum + Bacillus megatherium

\subsection{Experimental design}

The experimental treatments were a randomized complete block design with three replicates. The statistical analysis was carried out using the method of (Snedecor and Cochran, 1982).Means were compared by Duncan's multiple range test at $5 \%$ level of probability in the seasons of experiment.

\subsection{Data recorded}

The following data were recorded at the maturity stage, when $70 \%$ of the flowers bloomed and seeds started to be mature:

\subsubsection{Vegetative growth characters}

1- Plant height $(\mathrm{cm})$. 2-Number of branches/ plant. 3-Number of flowers / plant 4-Number of suckers/plant.5- Root length $(\mathrm{cm})$. 6- Aerial parts fresh and dry weights (g)/plant7- Flowers fresh and dry weight g/plant. 8- Roots fresh and dry weight $\mathrm{g} / \mathrm{plant}$. 
2.6.2. NPK content $\%$ of dry matter of herb, flower heads and roots according to the described methods of Cottenie et al., 1982).

2.6.3. Chicoric acid content (\% of dry matter) as a caffeic acid derivative according to (Bauer and Wagner, 1988).

\section{RESULTS AND DISCUSSION}

\subsection{Vegetative, flowering and rooting growth}

The data presented in Tables (3) and (4) show that, all organic manures with biofertilizers treatments significantly increased the vegetative, flowering and rooting growth parameters, and fresh and dry weights of Echinacea purpurea plant, compared with the chemical fertilizer treatment in the two seasons respectively.
Treating Echinacea purpurea plant with organic manure + a mixture of biofertilozers gave the highest vegetative, flowering and rooting growth parameters, and the highest fresh and dry weights per plant, followed by treatments fertilized with organic manure + one strain of biofertilizer. While the lowest vegetative, flowering and rooting growth parameters were occurred the chemical fertilizer treatment.

The highest values were 61.8 and $59.3 \mathrm{~cm}$ for plant height, 15.7 and 15.7 number of branches, 49.7 and 52.3 number of flowers, 7.67 and 7.00 number of suckers, 24.6 and $24.4 \mathrm{~cm}$ root length, , obtained from CH.M+ Azospirillum lipoferum + Bacillus megatherium in the two seasons respectively. The same results of organic

Table (3): Effect of organic manure with biofertilizer on vegetative,flowering and rooting growth of Echinacea purpurea plants in the first season 2005/2006.

\begin{tabular}{|c|c|c|c|c|c|c|c|c|c|c|c|}
\hline \multirow[b]{2}{*}{ Treatments } & \multirow{2}{*}{$\begin{array}{c}\text { Plant } \\
\text { height } \\
\text { cm }\end{array}$} & \multirow{2}{*}{$\begin{array}{c}\text { No. of } \\
\text { branches } \\
\text { /plant }\end{array}$} & \multirow{2}{*}{$\begin{array}{l}\text { No. of } \\
\text { flowers } \\
\text { /plant }\end{array}$} & \multirow{2}{*}{$\begin{array}{l}\text { No. of } \\
\text { suckers } \\
\text { /plant }\end{array}$} & \multicolumn{2}{|c|}{$\begin{array}{c}\text { Aerial } \\
\text { parts g/plant }\end{array}$} & \multicolumn{2}{|c|}{$\begin{array}{c}\text { Flowers weight } \\
\text { g/plant }\end{array}$} & \multirow{2}{*}{$\begin{array}{l}\text { Root } \\
\text { length }\end{array}$} & \multicolumn{2}{|c|}{$\begin{array}{l}\text { Roots weight } \\
\text { g/plant }\end{array}$} \\
\hline & & & & & $\begin{array}{c}\text { Fresh } \\
\text { wt. }\end{array}$ & Dry wt. & $\begin{array}{l}\text { Fresh } \\
\text { wt. }\end{array}$ & Dry wt. & & $\begin{array}{c}\text { Fresh } \\
\text { wt. }\end{array}$ & $\begin{array}{l}\text { Dry } \\
\text { wt. }\end{array}$ \\
\hline NPK & $42.2 \mathrm{e}$ & $11.7 \mathrm{c}$ & $37.7 \mathrm{e}$ & $5.0 \mathrm{c}$ & $104.9 \mathrm{a}$ & $30.4 \mathrm{c}$ & $150.3 \mathrm{e}$ & $40.8 \mathrm{f}$ & 18.0f & $32.4 \mathrm{e}$ & $10.5 \mathrm{~d}$ \\
\hline FYM+A & $43.6 \mathrm{e}$ & $12.3 \mathrm{c}$ & $43.0 \mathrm{c}$ & $5.67 \mathrm{c}$ & $125 \mathrm{a}$ & $38.5 \mathrm{abc}$ & $165.6 \mathrm{~d}$ & 42.2ef & $19.5 \mathrm{de}$ & $46.2 \mathrm{c}$ & $14.6 \mathrm{bc}$ \\
\hline FYM+B & $44.5 \mathrm{de}$ & $12.3 \mathrm{c}$ & $42.7 \mathrm{c}$ & $5.33 \mathrm{c}$ & $116.5 \mathrm{a}$ & $33.3 \mathrm{bc}$ & $158.3 \mathrm{de}$ & $42.3 \mathrm{ef}$ & 18.7ef & $40.9 d$ & $12.7 \mathrm{~cd}$ \\
\hline $\mathbf{F Y M}+\mathrm{A}+\mathrm{B}$ & $47.4 \mathrm{~d}$ & $14.3 b$ & $48.3 \mathrm{a}$ & 7.0ab & $126.5 \mathrm{a}$ & $38.3 \mathrm{abc}$ & $203.6 b$ & $55.4 \mathrm{bc}$ & $23.6 b$ & $53.7 \mathrm{ab}$ & $16.6 \mathrm{ab}$ \\
\hline $\mathrm{CHM}+\mathrm{A}$ & $53.0 \mathrm{c}$ & $12.3 \mathrm{c}$ & $41.0 \mathrm{~d}$ & $5.67 \mathrm{c}$ & $155 \mathrm{a}$ & $39.1 \mathrm{abc}$ & $211.0 \mathrm{~b}$ & $54.2 \mathrm{c}$ & $22.0 \mathrm{c}$ & $52.4 \mathrm{ab}$ & $16.1 \mathrm{ab}$ \\
\hline $\mathrm{CHM}+\mathrm{B}$ & $52.0 \mathrm{c}$ & $12.0 \mathrm{c}$ & $42.7 \mathrm{c}$ & $5.00 \mathrm{c}$ & $148.8 \mathrm{a}$ & $40.7 \mathrm{ab}$ & $200.0 b c$ & $50.3 d$ & $19.8 \mathrm{~d}$ & $52.0 \mathrm{ab}$ & $16.0 \mathrm{ab}$ \\
\hline $\mathrm{CHM}+\mathrm{A}+\mathrm{B}$ & $61.8 \mathrm{a}$ & $15.7 \mathrm{a}$ & $49.7 \mathrm{a}$ & $7.67 \mathrm{a}$ & $174.5 \mathrm{a}$ & $45.9 a$ & $234.0 \mathrm{a}$ & $58.9 \mathrm{a}$ & $24.6 \mathrm{a}$ & $56.9 \mathrm{a}$ & $17.3 \mathrm{a}$ \\
\hline Compost $+\mathbf{A}$ & $52.9 \mathrm{c}$ & $12.7 \mathrm{c}$ & $45.7 b$ & $5.67 \mathrm{c}$ & $135 \mathrm{a}$ & $38.5 \mathrm{abc}$ & $191.3 \mathrm{c}$ & $52.1 \mathrm{~cd}$ & $19.3 \mathrm{de}$ & $49.5 \mathrm{bc}$ & $15.9 \mathrm{ab}$ \\
\hline Compost+B & 45.4de & $12.0 \mathrm{c}$ & $38.3 \mathrm{e}$ & $6.67 b$ & $122.7 \mathrm{a}$ & $40.5 \mathrm{ab}$ & $164.3 \mathrm{~d}$ & $44.6 \mathrm{e}$ & $18.9 \mathrm{ef}$ & $39.6 \mathrm{~d}$ & $12.4 \mathrm{~cd}$ \\
\hline Compost $+A+B$ & $58.1 \mathrm{~b}$ & $15.3 \mathrm{ab}$ & $49.3 \mathrm{a}$ & $6.67 b$ & $157.7 \mathrm{a}$ & $44.7 \mathrm{ab}$ & $224.0 \mathrm{a}$ & $57.7 \mathrm{ab}$ & $24.5 \mathrm{a}$ & $54.2 \mathrm{ab}$ & $17.1 \mathrm{a}$ \\
\hline
\end{tabular}

Mean values followed by the same letter within the treatments are not significantly different at $5 \%$ according to Duncan's multiple range tests. NPK:Recommended dose FYM:Farm Yard Manure $\quad$ CHM: Chicken Manure $\quad$ A: Azospirillum linoferum $\quad$ B: Bacillus megatherium Compost: Nile Combost

Table (4): Effect of organic manure with biofertilizer and biofertilizer on vegetative, flowering and rooting growth of Echinacea purpurea plants in the second season 2006/2007.

\begin{tabular}{|c|c|c|c|c|c|c|c|c|c|c|c|}
\hline \multirow[b]{2}{*}{ Treatments } & \multirow{2}{*}{$\begin{array}{c}\text { Plant } \\
\text { height } \\
\text { cm }\end{array}$} & \multirow{2}{*}{$\begin{array}{l}\text { No. of } \\
\text { branches } \\
\text { /plant }\end{array}$} & \multirow{2}{*}{$\begin{array}{l}\text { No. of } \\
\text { flowers } \\
\text { /plant }\end{array}$} & \multirow{2}{*}{$\begin{array}{l}\text { No. of } \\
\text { suckers } \\
\text { /plant }\end{array}$} & \multicolumn{2}{|c|}{$\begin{array}{c}\text { Aerial } \\
\text { parts g/plant }\end{array}$} & \multicolumn{2}{|c|}{$\begin{array}{c}\text { Flowers weight } \\
\text { g/plant }\end{array}$} & \multirow{2}{*}{$\begin{array}{c}\text { Root } \\
\text { length } \\
\mathrm{cm}\end{array}$} & \multicolumn{2}{|c|}{$\begin{array}{c}\text { Roots weight } \\
\text { g/plant }\end{array}$} \\
\hline & & & & & $\begin{array}{c}\text { Fresh } \\
\text { wt. }\end{array}$ & $\begin{array}{c}\text { Dry } \\
\text { wt. }\end{array}$ & $\begin{array}{l}\text { Fresh } \\
\text { wt. }\end{array}$ & $\begin{array}{c}\text { Dry } \\
\text { wt. }\end{array}$ & & $\begin{array}{c}\text { Fresh } \\
\text { wt. }\end{array}$ & $\begin{array}{l}\text { Dry } \\
\text { wt. }\end{array}$ \\
\hline NPK & $41.17 \mathrm{e}$ & $11.00 \mathrm{~d}$ & $37.7 \mathrm{e}$ & $4.67 \mathrm{~cd}$ & $110.4 \mathrm{f}$ & $26.7 d$ & $144.7 \mathrm{~h}$ & $36.6 \mathrm{f}$ & $17.4 \mathrm{~d}$ & $29.4 \mathrm{f}$ & $7.82 \mathrm{e}$ \\
\hline FYM+A & $43.0 \mathrm{De}$ & $11.33 c$ & $40.3 \mathrm{~d}$ & $5.00 \mathrm{bcd}$ & $120.7 \mathrm{e}$ & $28.6 \mathrm{~d}$ & $162.6 f$ & $40.2 \mathrm{e}$ & $18.4 \mathrm{~cd}$ & $43.2 \mathrm{~cd}$ & $9.69 \mathrm{~d}$ \\
\hline FYM+B & 43.5De & $11.00 \mathrm{c}$ & $37.00 \mathrm{e}$ & $4.33 \mathrm{~d}$ & $123.5 \mathrm{e}$ & $37.4 \mathrm{~b}$ & $155.3 \mathrm{~g}$ & $39.1 \mathrm{ef}$ & $18.4 \mathrm{~cd}$ & $37.9 \mathrm{de}$ & $12.9 b c$ \\
\hline $\mathbf{F Y M}+\mathbf{A}+\mathbf{B}$ & $47.3 \mathrm{~cd}$ & $11.33 \mathrm{c}$ & $48.7 b$ & $6.00 \mathrm{abc}$ & $133.3 d$ & $36.8 b$ & $201.6 \mathrm{~d}$ & $52.3 b c$ & $22.3 b$ & $49.4 \mathrm{ab}$ & $13.7 \mathrm{ab}$ \\
\hline $\mathbf{C H M + A}$ & $52.5 b$ & $11.7 \mathrm{c}$ & $45.0 \mathrm{c}$ & $5.00 \mathrm{bcd}$ & $151.7 \mathrm{~b}$ & $36.4 \mathrm{~b}$ & $207.3 c$ & $51.2 \mathrm{c}$ & $21.00 \mathrm{~b}$ & $49.4 \mathrm{ab}$ & $13.6 \mathrm{ab}$ \\
\hline CHM+B & $51.3 b c$ & $13.7 b$ & $42.0 \mathrm{~d}$ & $4.33 \mathrm{~d}$ & $144.4 \mathrm{c}$ & $35.4 \mathrm{bc}$ & $198.8 \mathrm{~d}$ & $46.9 \mathrm{~d}$ & $19.2 \mathrm{c}$ & 49.0abc & $13.1 \mathrm{abc}$ \\
\hline $\mathrm{CHM}+\mathrm{A}+\mathbf{B}$ & $59.3 \mathrm{a}$ & $15.7 \mathrm{a}$ & $52.3 \mathrm{a}$ & $7.00 \mathrm{a}$ & $169.2 \mathrm{a}$ & $43.6 \mathrm{a}$ & $230.0 \mathrm{a}$ & $55.9 \mathrm{a}$ & $24.4 \mathrm{a}$ & $53.9 \mathrm{a}$ & $15.0 \mathrm{a}$ \\
\hline Compost $+A$ & $52.0 \mathrm{~b}$ & $11.7 \mathrm{c}$ & $42.3 \mathrm{~d}$ & $5.00 \mathrm{bcd}$ & $126.3 \mathrm{e}$ & $34.3 b c$ & $186.2 \mathrm{e}$ & $50.8 \mathrm{c}$ & $18.7 \mathrm{~cd}$ & $46.5 b c$ & $11.6 \mathrm{c}$ \\
\hline Compost+B & 44.7de & $12.00 \mathrm{c}$ & $42.0 \mathrm{~d}$ & $6.00 \mathrm{abc}$ & $120.7 \mathrm{e}$ & $31.0 \mathrm{~cd}$ & $161.4 \mathrm{f}$ & $41.6 \mathrm{e}$ & $18.3 \mathrm{~cd}$ & $36.6 \mathrm{e}$ & 9.4de \\
\hline Compost $+A+B$ & $57.7 \mathrm{a}$ & $15.33 \mathrm{a}$ & $49.0 \mathrm{~b}$ & $6.33 \mathrm{ab}$ & $153.3 b$ & $42.2 \mathrm{a}$ & $222.2 b$ & $54.7 \mathrm{ab}$ & $24.00 \mathrm{a}$ & $51.2 \mathrm{ab}$ & $14.8 \mathrm{ab}$ \\
\hline
\end{tabular}


fertilization treatment have been reported by ElGengaihi and Abdallah (1982), El-Sayed (1985) and $\mathrm{Li}$, (1996).

\section{2. $N, P$ and $K$ content}

The obtained data in Tables (5) and (6) indicate that organic manure + a mixture of biofertilizers treatment led to a significant increment in $\mathrm{N}, \mathrm{P}$ and $\mathrm{K} \%$ content compared with the other treatments under the same organic manure in the two seasons. strain of biofertilizer. While the lowest values of $\mathrm{N} \mathrm{P}$ and $\mathrm{K} \%$ content were obtained by treating plants with chemical fertilizer alone.

The highest values of nitrogen were 2.19 and $2.19 \%$ in herb, 2.52 and $2.48 \%$ in flower heads and 2.89 and $2.91 \%$ in roots. Also the highest values of phosphorous in herb were 0.39 and $0.35 \%$, in flower heads 0.42 and $0.39 \%$ and 0.62 and $0.59 \%$ in roots and the highest values of potassium were 3.32 and $3.31 \%$ in herb, 3.52 and

Table (5): Effects of organic manure with biofertilizer on $N, P$ and $K$ content $\%$ in different plant

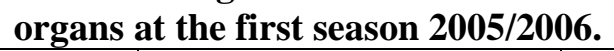

\begin{tabular}{|c|c|c|c|c|c|c|c|c|c|}
\hline \multirow{2}{*}{ Treatments } & \multicolumn{4}{|c|}{ Herb } & \multicolumn{3}{c|}{ Flower Heads } & \multicolumn{3}{c|}{ Roots } \\
\cline { 2 - 10 } & $\mathbf{\% N}$ & $\mathbf{\%} \mathbf{P}$ & $\mathbf{\%}$ & $\mathbf{\% N}$ & $\mathbf{\%} \mathbf{P}$ & $\mathbf{\%}$ & $\mathbf{\%}$ & $\mathbf{\%} \mathbf{P}$ & $\mathbf{\% K}$ \\
\hline NPK & $1.66 \mathrm{f}$ & $0.31 \mathrm{c}$ & $2.25 \mathrm{~h}$ & $1.84 \mathrm{f}$ & $0.32 \mathrm{~d}$ & $3.16 \mathrm{~d}$ & $2.73 \mathrm{~cd}$ & $0.50 \mathrm{cde}$ & $1.42 \mathrm{e}$ \\
\hline FYM+A & $1.67 \mathrm{f}$ & $0.33 \mathrm{bc}$ & $2.53 \mathrm{fF}$ & $1.88 \mathrm{f}$ & $0.34 \mathrm{bcd}$ & $3.18 \mathrm{~d}$ & $2.74 \mathrm{c}$ & $0.47 \mathrm{de}$ & $1.44 \mathrm{de}$ \\
\hline FYM+B & $1.81 \mathrm{e}$ & $0.29 \mathrm{c}$ & $2.33 \mathrm{~g}$ & $2.06 \mathrm{~d}$ & $0.33 \mathrm{~cd}$ & $3.41 \mathrm{~b}$ & $2.66 \mathrm{e}$ & $0.44 \mathrm{e}$ & $1.46 \mathrm{cde}$ \\
\hline FYM+A+B & $2.03 \mathrm{c}$ & $0.35 \mathrm{abc}$ & $2.96 \mathrm{~cd}$ & $2.14 \mathrm{c}$ & $0.37 \mathrm{abcd}$ & $3.37 \mathrm{bc}$ & $2.76 \mathrm{bc}$ & $0.53 \mathrm{bc}$ & $1.47 \mathrm{cde}$ \\
\hline $\mathbf{C H M + A}$ & $1.94 \mathrm{~d}$ & $0.34 \mathrm{abc}$ & $3.00 \mathrm{c}$ & $2.23 \mathrm{~b}$ & $0.39 \mathrm{abc}$ & $3.36 \mathrm{bc}$ & $2.76 \mathrm{bc}$ & $0.55 \mathrm{bc}$ & $1.51 \mathrm{bc}$ \\
\hline $\mathbf{C H M + B}$ & $1.93 \mathrm{~d}$ & $0.30 \mathrm{c}$ & $2.91 \mathrm{~d}$ & $2.07 \mathrm{~d}$ & $0.34 \mathrm{bcd}$ & $3.21 \mathrm{~d}$ & $2.66 \mathrm{e}$ & $0.52 \mathrm{bcd}$ & $1.49 \mathrm{bcd}$ \\
\hline CHM+A+B & $2.19 \mathrm{a}$ & $0.39 \mathrm{a}$ & $3.32 \mathrm{a}$ & $2.52 \mathrm{a}$ & $0.42 \mathrm{a}$ & $3.52 \mathrm{a}$ & $2.89 \mathrm{a}$ & $0.62 \mathrm{a}$ & $1.65 \mathrm{a}$ \\
\hline Compost+A & $1.83 \mathrm{e}$ & $0.33 \mathrm{bc}$ & $2.75 \mathrm{e}$ & $2.04 \mathrm{~d}$ & $0.34 \mathrm{bcd}$ & $3.36 \mathrm{bc}$ & $2.74 \mathrm{c}$ & $0.51 \mathrm{bcd}$ & $1.46 \mathrm{cde}$ \\
\hline Compost+B & $1.79 \mathrm{e}$ & $0.30 \mathrm{c}$ & $2.27 \mathrm{gh}$ & $1.98 \mathrm{e}$ & $0.37 \mathrm{abcd}$ & $3.34 \mathrm{c}$ & $2.68 \mathrm{de}$ & $0.53 \mathrm{bc}$ & $1.47 \mathrm{cde}$ \\
\hline Compost+A+B & $2.11 \mathrm{~b}$ & $0.37 \mathrm{ab}$ & $3.25 \mathrm{~b}$ & $2.24 \mathrm{~b}$ & $0.40 \mathrm{ab}$ & $3.49 \mathrm{a}$ & $2.81 \mathrm{~b}$ & $0.57 \mathrm{ab}$ & $1.54 \mathrm{~b}$ \\
\hline
\end{tabular}

Mean values followed by the same letter within the treatments are not significantly different at 5\% according to Duncan's multiple range tests. NPK:Recommended dose FYM:Farm Yard Manure CHM: Chicken Manure A: Azospirillum lipoferum

Table (6): Effects of organic manure with biofertilizer on N, P and $K$ content \%in different plant organs at the second season 2006/2007.

\begin{tabular}{|c|c|c|c|c|c|c|c|c|c|}
\hline \multirow{2}{*}{ Treatments } & \multicolumn{4}{|c|}{ Herb } & \multicolumn{3}{c|}{ flower Heads } & \multicolumn{3}{c|}{ Roots } \\
\cline { 2 - 10 } & $\mathbf{\% N}$ & $\mathbf{\%} \mathbf{P}$ & $\mathbf{\% K}$ & $\mathbf{\% N}$ & $\mathbf{\%} \mathbf{P}$ & $\mathbf{\% K}$ & $\mathbf{\% N}$ & $\mathbf{\%} \mathbf{P}$ & $\mathbf{\% K}$ \\
\hline NPK & $1.63 \mathrm{f}$ & $0.27 \mathrm{~d}$ & $2.14 \mathrm{~h}$ & $1.82 \mathrm{~g}$ & $0.31 \mathrm{~b}$ & $3.14 \mathrm{f}$ & $2.62 \mathrm{f}$ & $0.44 \mathrm{~d}$ & $1.43 \mathrm{~d}$ \\
\hline FYM+A & $1.65 \mathrm{f}$ & $0.28 \mathrm{~cd}$ & $2.49 \mathrm{f}$ & $2.06 \mathrm{~cd}$ & $0.31 \mathrm{~b}$ & $3.16 \mathrm{ef}$ & $2.69 \mathrm{de}$ & $0.51 \mathrm{bc}$ & $1.45 \mathrm{~cd}$ \\
\hline FYM+B & $1.80 \mathrm{e}$ & $0.31 \mathrm{bcd}$ & $2.28 \mathrm{~g}$ & $1.91 \mathrm{f}$ & $0.34 \mathrm{~b}$ & $3.39 \mathrm{c}$ & $2.65 \mathrm{ef}$ & $0.45 \mathrm{~cd}$ & $1.4 \mathrm{~cd}$ \\
\hline FYM+A+B & $2.01 \mathrm{c}$ & $0.34 \mathrm{ab}$ & $2.96 \mathrm{~cd}$ & $2.11 \mathrm{c}$ & $0.35 \mathrm{~b}$ & $3.36 \mathrm{~cd}$ & $2.70 \mathrm{de}$ & $0.52 \mathrm{~b}$ & $1.48 \mathrm{bcd}$ \\
\hline $\mathbf{C H M + A}$ & $1.89 \mathrm{~d}$ & $0.33 \mathrm{abc}$ & $2.10 \mathrm{c}$ & $2.22 \mathrm{~b}$ & $0.35 \mathrm{~b}$ & $3.35 \mathrm{~cd}$ & $2.76 \mathrm{bc}$ & $0.52 \mathrm{~b}$ & $1.50 \mathrm{bc}$ \\
\hline $\mathbf{C H M + B}$ & $1.87 \mathrm{~d}$ & $0.28 \mathrm{~cd}$ & $2.90 \mathrm{~d}$ & $2.06 \mathrm{~cd}$ & $0.33 \mathrm{~b}$ & $3.21 \mathrm{e}$ & $2.65 \mathrm{ef}$ & $0.51 \mathrm{bc}$ & $1.49 \mathrm{bcd}$ \\
\hline CHM+A+B & $2.19 \mathrm{a}$ & $0.35 \mathrm{a}$ & $3.31 \mathrm{a}$ & $2.48 \mathrm{a}$ & $0.39 \mathrm{a}$ & $3.61 \mathrm{a}$ & $2.91 \mathrm{a}$ & $0.59 \mathrm{a}$ & $1.69 \mathrm{a}$ \\
\hline Compost+A & $1.80 \mathrm{e}$ & $0.32 \mathrm{bcd}$ & $2.74 \mathrm{e}$ & $2.01 \mathrm{de}$ & $0.33 \mathrm{~b}$ & $3.34 \mathrm{~cd}$ & $2.72 \mathrm{~cd}$ & $0.51 \mathrm{bc}$ & $1.47 \mathrm{~cd}$ \\
\hline Compost+B & $1.76 \mathrm{e}$ & $0.30 \mathrm{bcd}$ & $2.24 \mathrm{~g}$ & $1.96 \mathrm{e}$ & $0.32 \mathrm{~b}$ & $3.30 \mathrm{~d}$ & $2.69 \mathrm{de}$ & $0.49 \mathrm{bcd}$ & $1.46 \mathrm{~cd}$ \\
\hline Compost+A+B & $2.09 \mathrm{~b}$ & $0.35 \mathrm{ab}$ & $3.24 \mathrm{~b}$ & $2.21 \mathrm{~b}$ & $0.37 \mathrm{ab}$ & $3.48 \mathrm{~b}$ & $2.81 \mathrm{~b}$ & $0.53 \mathrm{~b}$ & $1.52 \mathrm{~b}$ \\
\hline
\end{tabular}

Mean values followed by the same letter within the treatments are not significantly different at $5 \%$ according to Duncan's multiple range test. NPK:Recommended dose FYM:Farm Yard Manure Manure A: Azospirillum lipoferum $\quad$ B: Bacillus megatherium $\quad$ Compost: Nile Compost

CHM: Chicken

Treating Echinacea purpurea plants with organic manure + a mixture of biofertilizers gave the highest values of $\mathrm{N}, \mathrm{P}$, and $\mathrm{K} \%$ content per plant followed by organic manure treatment + one
$3.61 \%$ in flower heads, 1.65 and $1.69 \%$ in roots (\% of dry matter in the two seasons, respectively).

3.3. Chicoric acid content (as a caffiec acid derivative) $\%$ of dry matters 
The data recorded in Table (7) show that all treatments fertilized by organic manure + a mixture of biofertilizers gave a significant increment of chicoric acid as compared with the same organic manure with one strain of biofertilizer in most cases in the two seasons.
Badran (2002) on cumin, Sha'alan (2005) on Nigella sativa, Abd El Aziz et al., (2007) on Rosmarinus officinalis, Azzaz et al. (2009) on fennel, and Hendawy and El Gengaihi (2010) on Borago officinalis and Echium vulgare.

\section{REFERENCES}

Table (7): Effect of organic manure with biofertilzer on chicoric acid content (\%) at the two seasons 2005/2006 and 2006/2007.

\begin{tabular}{|c|c|c|c|c|c|c|}
\hline \multirow{2}{*}{ Treatments } & \multicolumn{2}{|c|}{ Herb } & \multicolumn{2}{c|}{ Flower Heads } & \multicolumn{2}{c|}{ Roots } \\
\cline { 2 - 7 } & $\begin{array}{c}\text { First } \\
\text { season }\end{array}$ & $\begin{array}{c}\text { Second } \\
\text { season }\end{array}$ & $\begin{array}{c}\text { First } \\
\text { season }\end{array}$ & $\begin{array}{c}\text { Second } \\
\text { season }\end{array}$ & $\begin{array}{c}\text { First } \\
\text { season }\end{array}$ & $\begin{array}{c}\text { Second } \\
\text { season }\end{array}$ \\
\hline NPK & $1.13 \mathrm{~d}$ & $1.09 \mathrm{~d}$ & $1.863 \mathrm{e}$ & $1.86 \mathrm{~g}$ & $1.00 \mathrm{Fg}$ & $1.00 \mathrm{f}$ \\
\hline FYM+A & $1.15 \mathrm{~d}$ & $1.11 \mathrm{~d}$ & $1.92 \mathrm{de}$ & $1.92 \mathrm{ef}$ & $1.07 \mathrm{de}$ & $1.04 \mathrm{ef}$ \\
\hline FYM+B & $1.14 \mathrm{~d}$ & $1.11 \mathrm{~d}$ & $1.92 \mathrm{de}$ & $1.91 \mathrm{fg}$ & $0.96 \mathrm{~g}$ & $1.00 \mathrm{f}$ \\
\hline FYM+A+B & $1.21 \mathrm{bc}$ & $1.19 \mathrm{bc}$ & $1.99 \mathrm{c}$ & $1.96 \mathrm{de}$ & $1.13 \mathrm{bc}$ & $1.13 \mathrm{~cd}$ \\
\hline CHM+A & $1.25 \mathrm{~b}$ & $1.24 \mathrm{~b}$ & $2.016 \mathrm{ab}$ & $2.04 \mathrm{abc}$ & $1.17 \mathrm{~b}$ & $1.22 \mathrm{~b}$ \\
\hline CHM+B & $1.21 \mathrm{bc}$ & $1.20 \mathrm{bc}$ & $1.973 \mathrm{bc}$ & $2.01 \mathrm{bcd}$ & $1.11 \mathrm{~cd}$ & $1.1 \mathrm{bc}$ \\
\hline CHM+A+B & $1.33 \mathrm{a}$ & $1.30 \mathrm{a}$ & $2.06 \mathrm{a}$ & $2.08 \mathrm{a}$ & $1.30 \mathrm{a}$ & $1.29 \mathrm{a}$ \\
\hline Compost+A & $1.21 \mathrm{bc}$ & $1.20 \mathrm{bc}$ & $2.06 \mathrm{bc}$ & $2.00 \mathrm{~d}$ & $1.08 \mathrm{cde}$ & $1.09 \mathrm{de}$ \\
\hline Compost+B & $1.18 \mathrm{~cd}$ & $1.18 \mathrm{c}$ & $2.016 \mathrm{~cd}$ & $1.98 \mathrm{~cd}$ & $1.03 \mathrm{ef}$ & $1.05 \mathrm{ef}$ \\
\hline Compost+A+B & $1.24 \mathrm{~b}$ & $1.21 \mathrm{bc}$ & $2.103 \mathrm{ab}$ & $2.06 \mathrm{ab}$ & $1.18 \mathrm{~b}$ & $1.21 \mathrm{~b}$ \\
\hline
\end{tabular}

Mean values followed by the same letter within the treatments are not significantly different at 5\% according to Duncan's multiple range test. NPK:Recommended dose FYM:Farm Yard Manure CHM: Chicken Manure A: Azospirillum lipoferum B: Bacillus megatherium

Compost: Nile Compost

The highest values of chicoric acid occurred by fertilizing plants with organic manure + a mixture of biofertilizers, but the lowest values of chicoric acid per plant occurred with chemical fertilizer treatment.

Organic fertilization had a positive effect on chicoric acid content of the plants fertilized by chicken manure with a mixture of the used strains. Results obtained were significant in the two seasons; Chicoric content was 1.33 and 1.30 in herb, 2.06 and 2.08 in flower heads, 1.30 and 1.29 $\%$ of dry matter in roots in the two seasons, respectively.

These results may be due to the great benefits of organic manure with biofertilizers on amending the plants with their requirements of nutrients.

Organic manure supply plants with continuous nutrients which improve some physical properties of the soil and increase water retention than that for chemical fertilizers (Abd Elmoez et al., 1995); Filessbach et al., 2000). In addition, biofertilizers increase the availability of nutrients and provide plants with promoting substances such as indole acetic acid and gibberellins (Fayez et al., 1985).

The same effect of using organic manure + biofertilizers on other medicinal and aromatic plants was emphasized by Osman (2000) on coriander, Kandil, (2002) on fennel, Safwat and
Abd El Aziz M., Pokluda R., Abd El Wahab M. (2007).Influence of compost, microorganisms and NPK fertilizer upon growth, chemical composition and essential oil production of Rosmarinus officinalis, L. Not. Bot. Hort. Agrobat. Cluj, volume 35, Issue 1.

Abd Elmoez M.R., Ghali M.H., and Abd El Fatah. A. (1995). Conditioning of sand soil by organic wastes and its impact on $\mathrm{N}$. concentration and yield of broad bean. Zagazig J.Agric. Res, 22 (11): 221-233.

Abou El Fadl, I.A., Abou, Baker M. and El-Gamal A. M. (1990). Effect of different organic manure composts on Roselle (Hibiscus sabdariffa L.) plants and soil characteristics. Agic. Res. Review, 68 (5): 1077 -1087.

Azzaz, N.A., Hassan E.A. and Hamad E.H. (2009). The chemical constituent and vegetative and yielding characteristics of fennel plants treated with organic and biofertilizer instead of mineral fertilizer. Australian Journal of Basic and Applied Sciences, 3 (2): 579-587.

Badran, F.S. and Safwat M.S. (2004). Response of fennel plants to organic manure and biofertilizers in replacement of chemical fertilization. Egypt. J.Agric. Res., 82 (2): 247652. 
Barrett B. (2003). Medicinal properties of Echinacea: A critical review, Phytomedicine 10: 66-86.

Bauer R. and Wagner H. (1988). Echinacea der Sonnehut stand der for schung Zeitschriftfier der Phytotherapie, 9: 151-159.

Cottenie, A., Verloo M., Velghe M. and Camerlynck R. (1982). Chemical analysis of plant and soil. Laboratory of Analytical and Agrochemistry. State Univ. Ghent, Belgium. Http://Libdoc.who.int.

El-Gengaihi S., Abdallah N. and Sidrak I. (1982).The effect of fertilization levels on flowering, oleanolic acid and phytosterol content of Calendula officinalis L. Pharmazie, 37 (7): 511- 514.

El-Sayed A. A. (1985). Effect of nitrogen fertilization and some growth regulators on yarrow plants (Achillea millefoleium L.). M. Sc. Thesis, Fac. of Agric., Minia Univ.

Fayez M., Emam N.F, and Makboul H.E. (1985). The possible use of nitrogen fixing Azospirillum as biofertilizer for wheat plants. Egypt. J. Microbial., 20 (2): 199 -206.

Fliessbach A., Mader P., Dubois D. and Gunst L. (2000). Results from a 21 year old field trial. Organic farming enhances soil fertility and biodivesity. Bull. Res. Inst. Organic Agric., 1: 15- 19.

Hendawy S.F. (1995). Agriculture and chemical studies on Echinacea purpurea species. M.Sc. Thesis, Fac. Agric., Zagazig Univ, Egypt.

Hendawy, S.F. and El-Gengaihi S. (2010), Comparative response of Borago officinalis and Echium vulgare to different nitrogen and phosphorus sources. Journal of Herbs, Spices and Medicinal Plants, 16, (1) : 12-23.

Kandil M.A.M.H. (2002). The effect of fertilizers for conventional and organic farming on yield and oil quality of fennel (Foeniculum vulgare, Mill.) in Egypt. Ph.D. Thesis. Fakultat der echischen F., Carlo Wilhelmina Univ.

Kindscher, K. (1989). Ethno botany of purple coneflower Echinacea angustifolia Asteraceae and other Echinacea species. Economic Botany, 43: 498-507.

Li T. S. C. (1996). Nutrient weeds as soil amendment for organically grown herbs. Journal of Herbs, Spices and Medicinal Plants, 4:3-8.

Osman Y.A.H. (2000). Possibility of production of coriander (Coriandrum sativum, L.) under Sinai conditions. Ph.D. Thesis, Faculty of Agriculture, Cairo University, Egypt.

Rashad S. H. (1999). Effect of some agricultural practicies on the growth, yield and active ingredients of Echinacea plant "Echinacea purpurea". M.Sc. Thesis, Fac. Agric, Cairo University. Egypt.

Safwat M.S., Badran F.S. and Zayed G.A. (2001). Response of anise plants to different organic and biofertilization treatments. The $10^{\text {th }}$ Conf. of Medicinal and Aromatic plants, Cairo, Egypt.

Safwat M.S. and Badran F.S. (2002). Efficiency of organic and biofertilizers, in comparison with chemical fertilization on growth, yield and essential oil of cumin plants. The $9^{\text {th }}$ Conf. of Medicinal and Aromatic Plants, Cairo, Egypt.

Sha`alan M.N. (2005). Influence of biofertilization and chicken manure on growth, yield and seeds quality of Nigella sativa, L. plants. Egypt, J. Agric. Res., 83 (2): 811-828.

Snedecor G. W. and Cochran W.G. (1982). Statistical Method. $7^{\text {th }}$ Ed., Iowa State Univ. Press, Ames, Iowa, USA. 
تأثير التسميد العضوى مع التسميد الحيوى على النمو والتزهير والمكونات الكيم يإئة فى نبات الاثنسيا بوريوريا

عزة محمد سعيد عرفة ـ هند مصطفي فهمي - مكارم عبداللاه محمل سعيد*- هالة صابر عبد ربه مشعل*

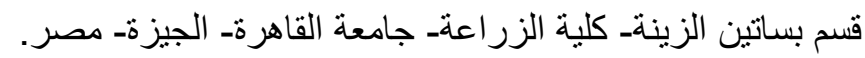

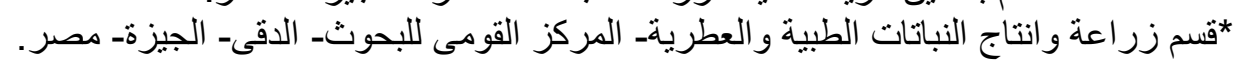

ملخص

أجريت هذه الدراسة خلال موسمى 2005 2006 2006 /2007 فى مشتل قسم بساتين الزينة ـ كلية الزراعة-

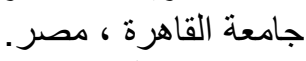

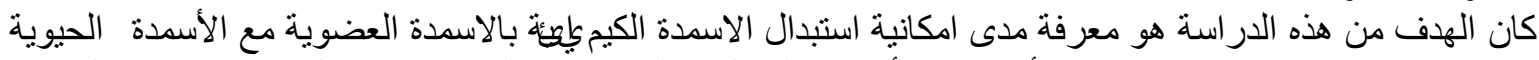

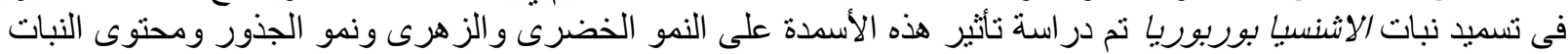

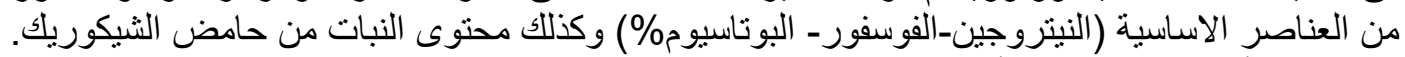

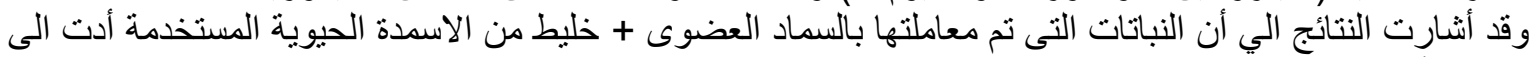

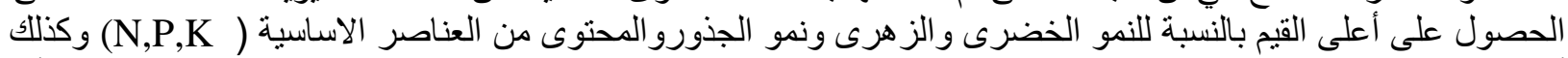

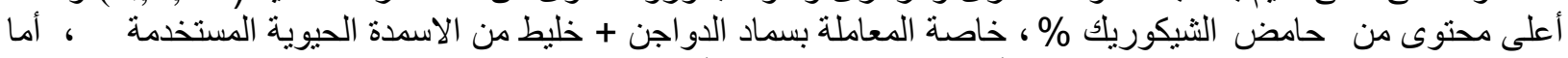

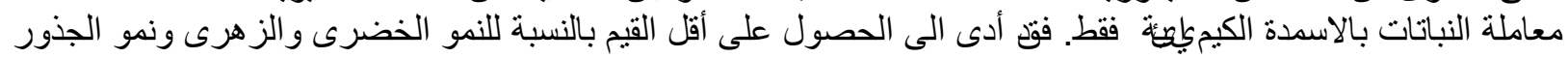

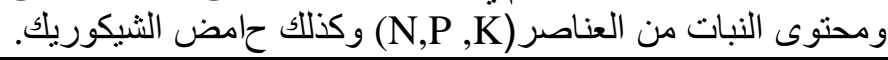

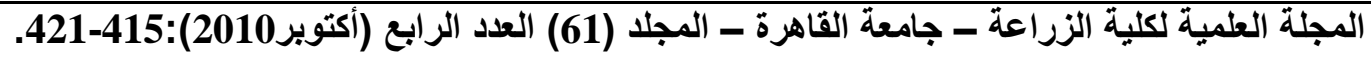

